cell and caused to separate into two fractions, one rich in solids, the other poor in solids content. The ratio of the concentrations (the separation factor) was observed to be 2.5. (higher factors should be obtainable with improved design).

4. Suspensions of several polar solids in less polar liquids were precipitated out using the effect of the nonuniform field.
5. Equations derived for the effect showed the force to depend upon the fifth power of the radius of the central electrode for spherical geometries, and upon the third power of the radius for cylindrical geometries. It was also shown that the velocity of a particle in such fields is proportional to the square of the particle radius, hence, the dependence noted above upon particle size.

\title{
Note on Diffusive Separation of Gas Mixtures in Flow Fields*
}

\author{
V. C. LIU \\ Engineering Research Institute, University of Michigan, Ann Arbor, Michigan
}

(Received February 10, 1958)

\begin{abstract}
This paper discusses the effect of pressure-diffusion flux upon the concentration distribution of gas mixtures in flow fields. The equation of concentration is formulated for a binary gas mixture in which the mass ratio is large and the concentration of the lighter gas is very small. An asymptotic solution to the steady-state equation of concentration is given for an irrotational and incompressible flow.

As an illustration, the diffusive separation (i.e., deviation from the original homogeneous state) of a mixture of helium and nitrogen along streamlines at the entrance to a long straight channel is calculated. It is assumed that the pressure inside the channel is $90 \%$ of that in the free stream, and that the diffusion coefficient of the mixture corresponds with the atmospheric conditions at $80-\mathrm{km}$ altitude.
\end{abstract}

\section{INTRODUCTION}

$\mathbf{I}^{\mathrm{c}}$ $\mathrm{N}$ Chapman's recent discussion ${ }^{1}$ of thermal diffusion in gases, he reviewed a newly proposed method by this author of separating gas mixtures via suction of the thermal-diffusion boundary layer along a heated surface (referring to an unpublished report ${ }^{2}$ ). A paper dealing with Part I of this report is now being published. ${ }^{3}$ The present paper summarizes Part II of the unpublished report which deals with mixture separation in a flow field due to existing pressure-diffusion flux.

It is well known that mixture flow of Knudsen gases is diffusively separative. As the mean free path relative to a characteristic dimension of the flow decreases, intermolecular collisions, which cause momentum transfer, become increasingly important. In a continuous medium, the flow is almost always nonseparative. The physical laws governing the intermediate range of flow remain the least known. The basic phenomenon of interaction between mass flow and pressure diffusion leading to diffusive separation of gas mixtures will be treated here.

\section{ANALYSIS}

Consider a binary mixture in which both the volume concentration of the lighter gas $n_{10}$ and the molecular

* This work is supported by the Meteorological Branch of Evans Signal Laboratory (Contract No. DA-36-039 SC-64659).

${ }^{1} \mathrm{~S}$. Chapman, Second Biennial Gas Dynamics Symposium, Tech. Inst. Northwestern University (August, 1957).

2 V. C. Liu, "Diffusive separation of gas mixtures in flow fields, parts I and II," Mich. Univ. Eng. Research Inst. Rept. 2387. -29-T (Ann Arbor, 1957).

${ }^{3}$ V. C. Liu, Quart. J. Mech. Appl. Math. (to be published). mass ratio $m_{1} / m_{2}$ are much less than unity. The equation governing $n_{10}$ in an incompressible and isothermal flow field can be formulated from the application of the equation of continuity to the total flow and to the light component flow. If we adopt the formula for diffusion flux given by Chapman and Cowling 4 and neglect the body force and thermal-diffusion terms, the diffusion equation in a two-dimensional flow field $(u, v) \dagger$ can be obtained.

$$
\begin{aligned}
u \frac{\partial n_{10}}{\partial x}+\frac{\partial n_{10}}{\partial y}= & D_{12}\left(\frac{\partial^{2}}{\partial x^{2}}+\frac{\partial^{2}}{\partial y^{2}}\right) n_{10} \\
& +D_{12}\left(\frac{\partial G}{\partial x} \frac{\partial n_{10}}{\partial x}+\frac{\partial G}{\partial y} \frac{\partial n_{10}}{\partial y}\right) \\
& +D_{12} n_{10}\left(\frac{\partial^{2} G}{\partial x^{2}}+\frac{\partial^{2} G}{\partial y^{2}}\right)
\end{aligned}
$$

where $m_{2} G=\left(m_{2}-m_{1}\right) \ln p$. Equation (1) can be simplified by transformation from the $x, y$ plane to the $\phi, \psi$ plane where $\phi$ denotes velocity potential $(u=\partial \phi / \partial x, v=\partial \phi / \partial y)$, and $\psi$, stream function $(u=\partial \psi / \partial y, v=-\partial \psi / \partial x)$. The transformed equation, after substitution of $n_{10}=N_{10} \exp \left[-\frac{1}{2}\left(G-\phi / D_{12}\right)\right]$,

4 S. Chapman and T. G. Cowling, The Mathematical Theory of Non-Uniform Gases (Cambridge University Press, New York, 1953), Eq. (7), p. 140

$\dagger$ The notation used in reference 4 is followed. 


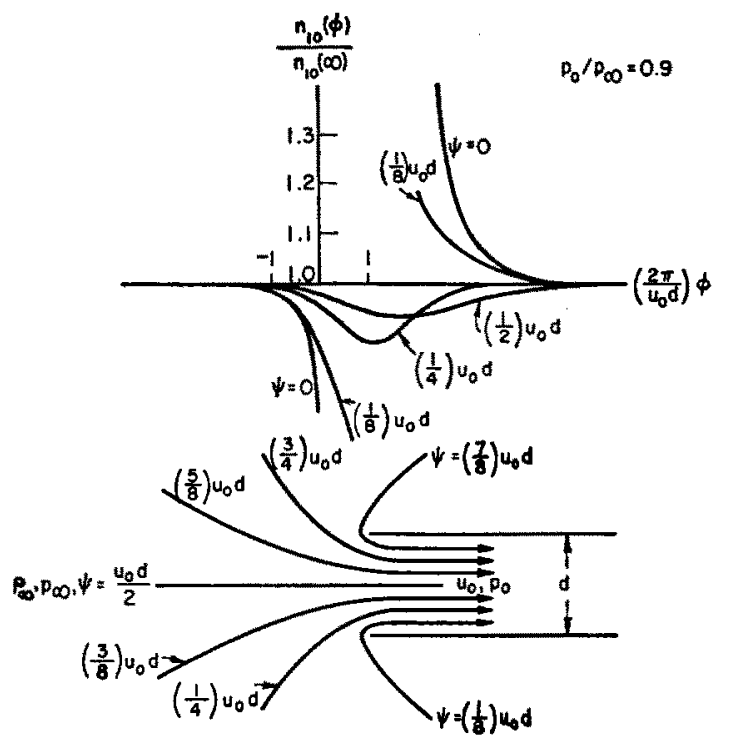

Frg. 1. Concentration distribution of the gas mixture, He and $\mathrm{N}_{2}$, along streamlines near the entrance to a long channel $\left(p_{\mathrm{a}} / p_{\infty}=0.9, D_{12}=9000 \mathrm{~cm}^{2} / \mathrm{sec}\right)$.

becomes

$$
\frac{\partial^{2} N_{10}}{\partial \phi^{2}}+\frac{\partial^{2} N_{10}}{\partial \psi^{2}}-F N_{10}=0
$$

where

$$
\begin{aligned}
F=\left(2 D_{12}\right)^{-2}-\left(2 D_{12}\right)^{-1} G_{\phi} & \\
& -\frac{1}{2}\left(G_{\phi \phi}+G_{\psi \psi}\right)+\frac{1}{4}\left(G_{\phi}{ }^{2}+G_{\psi}{ }^{2}\right) .
\end{aligned}
$$

The suffixes $\phi$ and $\psi$ denote $\partial / \partial \phi$ and $\partial / \partial \psi$, respectively.

Diffusive separation of an isothermal flow becomes important either when extremely high pressure gradient exists, such as in a shock-wave layer, or when $D_{12}$ of the gases is large, such as in a rarefied medium.

For concentration distribution along a streamline ( $\psi=$ const), Eq. (2) reduces to

$$
4 D_{12} \frac{d^{2} N_{10}}{d \phi^{2}}-\left(1-2 D_{12} G_{\phi}-2 D_{12}^{2} G_{\phi \phi}+D_{12}^{2} G_{\phi}^{2}\right) N_{10}=0 .
$$

For most physical problems with flows of continuous media, we have

$$
\left(2 D_{12}{ }^{2} G_{\phi \phi}-D_{12}{ }^{2} G_{\phi}{ }^{2}\right) \ll 2 D_{12} G_{\phi} \ll 1 .
$$

Equation (3), under condition (4), has an asymptotic solution of Green's type. ${ }^{5}$ In treating diffusive separation problems in which the initial value of concentration $N_{10}(\infty)$ corresponding to the undisturbed upstream condition is assumed constant, we have the concentration distribution $n_{10}(\phi)$ along a streamline ( $\psi=$ const $)$

$$
n_{10}(\phi) / n_{10}(\infty)=1+D_{12}(\partial G / \partial \phi)+\cdots .
$$

When the concentration distribution (or diffusive separation) at the entrance to an infinitely long channel is sought (see Fig. 1), we can calculate $G_{\phi}$ on the basis of irrotational flow theory of Borda's Mouthpiece, ${ }^{6}$ and thus obtain

$$
\left.\begin{array}{r}
\frac{n_{10}(\phi)}{n_{10}(\infty)}=\frac{2\left(m_{2}-m_{1}\right)}{m_{2}}\left(1-\frac{p_{o}}{p_{\infty}}\right)\left(\begin{array}{c}
\frac{\psi}{\delta}-e^{-\phi / \delta} \\
\delta
\end{array}\right) / \\
{\left[e^{\phi / \delta}+e^{-\phi / \delta}-2 \cos (\psi / \delta)\right],}
\end{array}\right\}
$$

where

$$
\delta=(d / 2 \pi)\left(2 p_{\infty} / \rho_{\infty}\right)^{\frac{1}{2}}\left(1-p_{0} / p_{\infty}\right)^{\frac{1}{2}} .
$$

Assuming, e.g., $p_{o} / p_{\infty}=0.9$ and $D_{12}=9000 \mathrm{~cm}^{2} / \mathrm{sec}$., the latter corresponding approximately to the atmospheric conditions at an altitude of $80 \mathrm{~km}$, we obtain the concentration distribution for a mixture of $\mathrm{He}$ and $\mathrm{N}_{2}$ as illustrated in Fig. 1. It is assumed that the ideal fluid theory gives a close approximation to the entrance flow of air to a channel at this altitude. ${ }^{7}$

Finally, the steady-state concentration (Fig. 1), although related to, should not be construed as the concentration of the intake mixture which is equal to the integrated transient fluxes of the gases.

\section{ACKNOWLEDGMENTS}

Stimulating discussions with Professor Sydney Chapman and assistance of Mr. Howard Jew in numerical computations are gratefully acknowledged.

${ }^{5} \mathrm{H}$. Jeffreys and B. S. Jeffreys, Mathematical Physics (Cambridge University Press, New York, 1956), pp. 522-528; for details see also H. Levy and E. A. Baggott, Numerical Solutions (Dover Publications, New York, 1950), pp. 230-232.

${ }^{6} \mathrm{~L}$. Prandtl and O. G. Tietjens, Fundamentals of Hydro- and Aeromechanics, (McGraw-Hill Book Company, Inc., New York, 1934), pp. 178-183.

${ }^{7}$ V. C. Liu, J. Geophys. Research 61, 171 (1956). 